\title{
Phase matched parametric amplification via four- wave mixing in optical microfibers
}

\author{
Muhammad I.M. Abdul Khudus, ${ }^{1,2,}{ }^{*}$ Francesco De lucia, ${ }^{1}$ Costantino \\ Corbari, ${ }^{1,3}$ Timothy Lee, ${ }^{1}$ Peter Horak, ${ }^{1}$ Pier Sazio, ${ }^{1}$ and Gilberto \\ BRAMBILLA ${ }^{1}$ \\ ${ }^{1}$ Optoelectronics Research Center, University of Southampton, Southampton, SO17 1BJ United Kingdom \\ 2 Photonics Research Centre, Department of Physics, Faculty of Science, University of Malaya, 50603 Kuala Lumpur, Malaysia \\ ${ }^{3}$ Renishaw plc, New Mills, Wotton-under-Edge, Gloucestershire, GL12 8JR United Kingdom \\ *Corresponding author: miak2g12@soton.ac.uk
}

Received XX Month XXXX; revised XX Month, XXXX; accepted XX Month XXXX; posted XX Month XXXX (Doc. ID XXXXX); published XX Month XXXX

\begin{abstract}
Four-wave mixing (FWM) based parametric amplification in optical microfibers (OMF) is demonstrated over a wavelength range of over $1000 \mathrm{~nm}$ by exploiting their tailorable dispersion characteristics to achieve phase matching. Simulations indicate that for any set of wavelengths satisfying the FWM energy conservation condition there are two diameters at which phase matching in the fundamental mode can occur. Experiments with a high-power pulsed source working in conjunction with a periodically poled silica fiber (PPSF), producing both fundamental and second harmonic signals, are undertaken to investigate the possibility of FWM parametric amplification in OMFs. Large increases of idler output power at the third harmonic wavelength were recorded for diameters close to the two phase matching diameters. A total amplification of more than $25 \mathrm{~dB}$ from the initial signal was observed in a $6 \mathrm{~mm}$ long optical microfiber, after accounting for the thermal drift of the PPSF and other losses in the system.
\end{abstract}

OCIS codes: (190.4370) Nonlinear optics, fibers; (190.4410) Nonlinear optics, parametric processes; (190.4380) Nonlinear optics, four-wave mixing;

http://dx.doi.org/10.1364/OL.99.099999

Four-wave mixing (FWM) is a process in which four waves interact via the optical Kerr nonlinearity of a medium, where two photons from one or two 'pump' waves (for instance at frequencies $\omega_{1}, \omega_{2}$ ) are annihilated, and two new photons, called the signal $\left(\omega_{3}\right)$ and idler $\left(\omega_{4}\right)$, are created [1]. One application of FWM is parametric amplification, where the power from one wavelength is transferred to another wavelength via the Kerr effect, which has been demonstrated in a variety of optical fibers, with the highest recorded conversion being $70 \mathrm{~dB}$ [1-4]. However, parametric amplification via FWM requires phase matching for efficient energy conversion in order to compensate for material and waveguide dispersion as well as nonlinear effects, often limiting the bandwidth of the process [5]. A number of schemes were proposed which allowed a broader bandwidth, but the largest realized bandwidth is $900 \mathrm{~nm}$ and $300 \mathrm{~nm}$ for solid core and microstructured optical fiber, respectively [6,7]. In this letter we demonstrate the possibility of using optical microfibers (OMFs) for FWM parametric amplification over a wavelength band of over $1000 \mathrm{~nm}$ by exploiting the tailorable dispersion characteristics of the OMF to optimize the diameter for phase matching. The technique, applicable for both parametric amplification as well as parametric conversion, theoretically allows for the amplification and generation of an almost arbitrary set of wavelengths, as well as the possibility of building fully fiberized light sources in the UV and mid-IR wavelength ranges.

OMFs are typically drawn from conventional fibers, most commonly by the flame brushing technique where a section of the fiber is heated to the softening point and pulled to reduce the diameter. By doing so, the optical core of the fiber gradually disappears, and the erstwhile cladding material effectively becomes the core, with air taking the role of the optical cladding. This allows for higher mode confinement, translating into an increased nonlinearity of up to 100 times the original value, as well as relatively low losses of typically $<1 \mathrm{~dB}$ [8]. The relatively high nonlinearity and tailorable dispersion has been previously exploited to achieve intermodal third harmonic generation in optical microfibers where energy transfer is achieved by parametric conversion from the fundamental mode at one frequency to a higher order mode at a higher frequency $[9,10]$, as well as an efficient means to achieve parametric conversion [11]. The phase matching and energy conservation conditions for efficient FWM, respectively, can be written as [5]:

$$
\begin{gathered}
\beta\left(\omega_{4}\right)+\beta\left(\omega_{3}\right)=\beta\left(\omega_{1}\right)+\beta\left(\omega_{2}\right) \\
\omega_{1}+\omega_{2}=\omega_{3}+\omega_{4}
\end{gathered}
$$

where the subscripts 1,2,3 and 4 refer to the four interacting wavelengths in the FWM process and $\beta(\omega)$ is the propagation constant for frequency $\omega$. 
In the case of degenerate FWM we have $\omega_{1}=\omega_{2}$. Here, we will assume that light at all wavelengths is propagating in the fundamental mode of the OMF as this would be the most efficient mode for FWM.

In order to find the OMF diameter required to achieve phase matching in Eq. (1), the rigorous eigenvalue equations for a step index refractive index profile were solved for a silica OMF. The wavelengths employed are harmonics of the fundamental at $1550 \mathrm{~nm}$ such that they automatically satisfy Eq. (2). To account for both degenerate and nondegenerate FWM, the wavelengths of the fundamental frequency (FF), second harmonic (SH), third harmonic $(\mathrm{TH})$ and fourth harmonic $(\mathrm{FH})$ were taken into consideration, as shown in Fig. 1a.

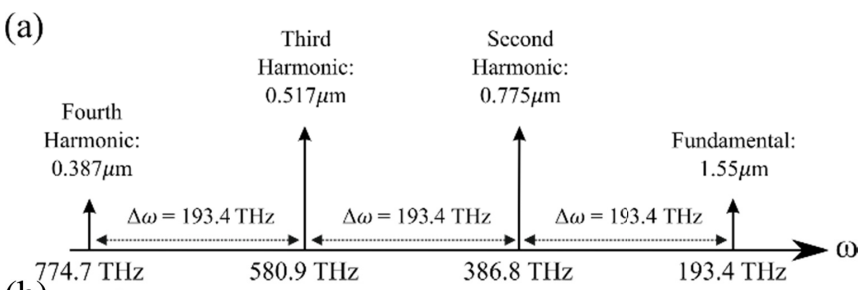

(b)

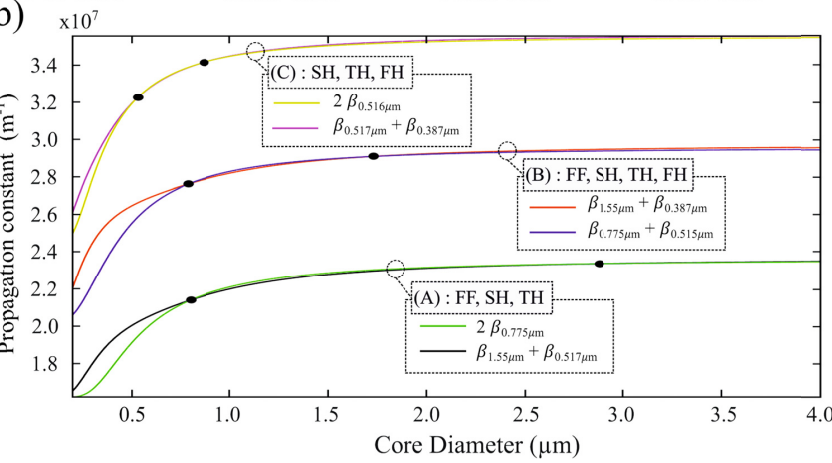

(c)

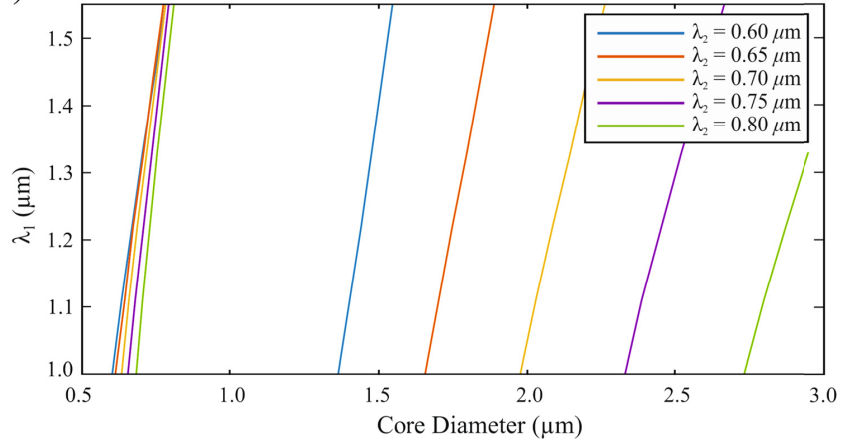

Fig. 1. (a) Frequencies investigated for FWM. The frequencies denoting pump, idler and signal vary with the scheme for FWM, as detailed in Table 1. (b) Relation between the OMF diameter and mode propagation constants for three different FWM schemes; black dots represent phase matching diameters satisfying Eq. (1). FF, SH, TH and FH represent the fundamental frequency, the second harmonic ( $\mathrm{SH}$ ), the third harmonic $(\mathrm{TH})$ and the fourth harmonic (FH), respectively. (c) Degenerate FWM phase matched diameter for a set first pump wavelength, $\lambda_{1}$, (left) and five different second pump wavelengths, $\lambda_{2}$. The idler wavelength $\lambda_{3}$ can be calculated from $1 / \lambda_{3}=2 / \lambda_{2}-1 / \lambda_{1}$. Two distinct sets of phase matching diameters can be discerned from the figure.

Three different FWM schemes were theoretically investigated, namely, FWM between (A) FF, SH and TH (degenerate); (B) FF, SH, TH and $\mathrm{FH}$ (non-degenerate); and (C) $\mathrm{SH}, \mathrm{TH}$ and $\mathrm{FH}$ (degenerate). The sum of the propagation constants on each side of Eq. (1) was plotted for each FWM scheme in Fig. 1b. The raw data used in this paper can be obtained from [12]. This yields the predicted phase matching diameters for the fundamental modes of the three FWM schemes discussed above. It is clear that for each FWM scheme, there are two phase matching diameters at which Eq. (1) is satisfied, denoted $d_{1}$ and $d_{2}$, as detailed in Table 1 . Experimentally, this translates into two different diameters at which FWM amplification is expected to occur in an OMF. Here, we shall only consider scheme (A), with schemes (B) and $(C)$ being studied in a future work. These wavelengths are chosen primarily for experimental reasons, but the technique can be easily extended to any number of wavelength combinations satisfying Eq. (2), as illustrated in Fig. 1c for degenerate FWM. It can be seen that phase matching can be achieved with a wide range of pump wavelengths $\left(\lambda_{1}\right.$ and $\left.\lambda_{2}\right)$, generating signals spanning from the visible to near IR wavelengths. A plot of the dispersion of the microfiber is shown in Fig. 2 to indicate the change of the dispersion with OMF diameter.

TABLE I: Phase matching diameters for the three different FWM schemes considered. $d_{1}$ and $d_{2}$ denote the first and second phase matching diameters, respectively.

\begin{tabular}{cccc}
\hline \hline \multicolumn{2}{c}{ FWM scheme and type } & Phase matching diameter $(\mu \mathrm{m})$ \\
& & $d_{1}$ & $d_{2}$ \\
\hline (A) Degenerate & 2.886 & 0.800 \\
(B) Non-degenerate & 1.719 & 0.790 \\
(C) & Degenerate & 0.872 & 0.530 \\
\hline \hline
\end{tabular}

To investigate this, a Master Oscillator Power Amplifier (MOPA) seeded by a narrowband continuous wave (CW) seed laser (Photonetics Tunics BT) working in the telecom C-band wavelength region (1530-1565 nm) was employed. The MOPA system (shown in Fig. 3) produced an output at a central wavelength of $1550.3 \mathrm{~nm}$ with a pulse width of $5 \mathrm{~ns}$, repetition rate of $200 \mathrm{kHz}$ and average powers of between $300 \mathrm{~mW}$ and $800 \mathrm{~mW}$, which translated into peak powers of between $300 \mathrm{~W}$ and $800 \mathrm{~W}$. The pulses were generated using an electro-optic modulator (EOM), which is then followed by an acoustooptic modulator (AOM) further along the chain to remove any interpulse amplified spontaneous emission (ASE) in the system to ensure that the majority of the power is contained in the pulse. Two fiberized thin film spectral filters operating in the C-band were also employed in order to improve the optical signal-to-noise ratio (OSNR) at the final output, required for efficient second harmonic generation. Polarization is managed by the use of two polarization controllers (PC) in the MOPA chain.

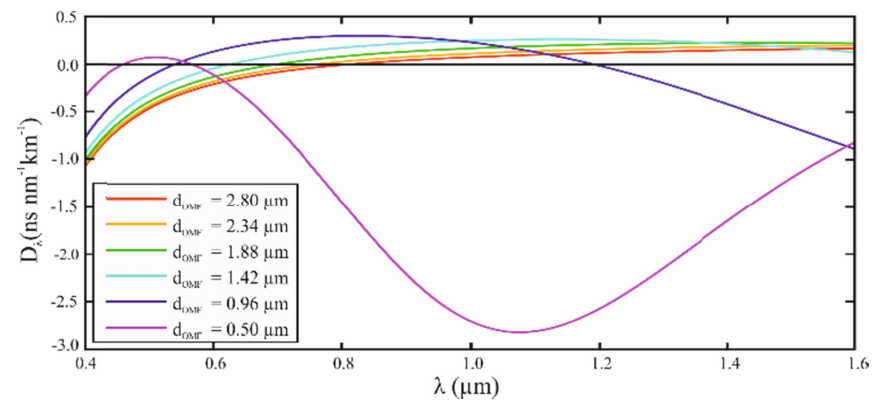

Fig. 2. Variation in the dispersion of the $\mathrm{OMF}\left(D_{\lambda}\right)$ with wavelength $\lambda$ for different OMF diameter $\left(d_{O M F}\right)$. It can be observed that the dispersion profile does not vary significantly for $d_{O M F}>1 \mu \mathrm{m}$, but changes significantly for diameters $0.5 \mu \mathrm{m}<d_{O M F}<1 \mu \mathrm{m}$.

The output of the MOPA was then connected to a periodically poled silica fiber (PPSF) designed to be quasi-phase matched (QPM) for efficient conversion of the FF at $1550.3 \mathrm{~nm}$ into the $\mathrm{SH}$ at $775.15 \mathrm{~nm}$ via second harmonic generation (SHG), which has a total insertion loss of $<0.5 \mathrm{~dB}$. The PPSF was fabricated from a twin-hole Germania doped fused silica fiber manufactured by Acreo Fiberlab. The two holes were 
first fully filled with Gallium liquid electrodes by means of a pressure filling technique similar to the one described in [13]. Tungsten wires were then inserted at each end of the two holes before the entire ensemble was sealed with superglue. The fiber was then thermally poled by applying a positive voltage of $7.5 \mathrm{kV}$ on both the electrodes whilst being heated at $250^{\circ} \mathrm{C}$ on a hotplate [14]. The sample was left to pole for $120 \mathrm{~min}$ before the hotplate was switched off and allowed to cool with the high voltage continually applied for a further $60 \mathrm{~min}$. This procedure induces an effective second order susceptibility $\chi_{e f f}^{(2)}$ uniformly across the silica fiber core by means of a third order rectification process expressed by the relationship $\chi_{\text {eff }}^{(2)} \approx 3 \chi^{(3)} E_{r e c}$, where $\chi^{(3)}$ is the third order susceptibility of silica and $E_{r e c}$ is the static frozen-in electric field due to poling $[15,16]$. Quasi-phase matching allows the wavevector mismatch between the fundamental and second harmonics to be compensated by modulating the nonlinearity induced by poling. This modulation is achieved by a periodic UV erasure of the poling field by local exposure to $355 \mathrm{~nm}$ radiation delivered as a $200 \mathrm{kHz}$ train of 8 ps pulses, focused to a 10 $\mu \mathrm{m} \times 100 \mu \mathrm{m}$ spot size with a total fluence of $200 \mathrm{~J} / \mathrm{cm}^{2}$ [17]. After exposing the poled fiber to this focused high-energy UV radiation, the resulting free carrier generation inside the fiber locally nullifies the frozen-in electric field $E_{\text {rec }}$ [18]. A spatially periodic exposure of the uniformly poled fiber therefore produces a modulation of $\chi_{\text {eff }}^{(2)}$. The period of this $\chi_{\text {eff }}^{(2)}$ grating is chosen to allow for QPM at the desired wavelength. The use of PPSF allows for the adoption of an all-fiber nonlinear device, and does not suffer from some constraints of the more common nonlinear devices such as thermal instabilities, relatively short interaction lengths, high costs, relatively low damage threshold, losses due to diffraction as well as from free space optical elements required for alignment and walk-off effects.

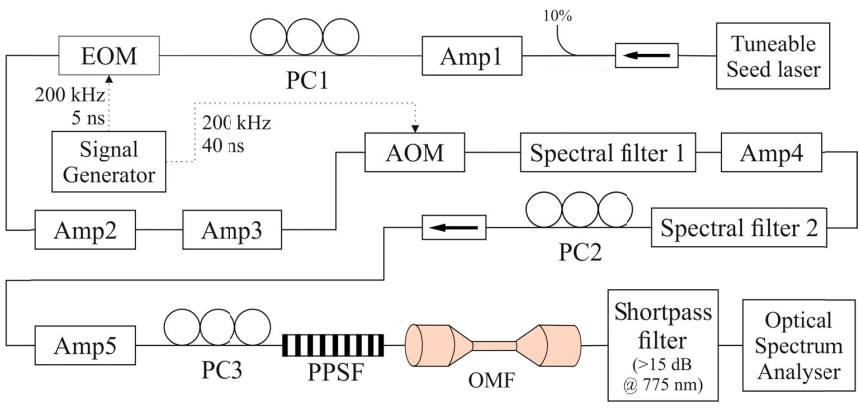

Fig. 3. Schematic of the experimental setup used to investigate enhancement of the idler signal due to phase matching in OMFs. A total of five amplifiers (Amp\#) were employed in conjunction with two spectral filters in order to minimize OSNR and inter-pulse ASE. Here, PPSF is a periodically poled silica fiber; PC\# is a polarization controller.

As the PPSF is highly sensitive to polarization, the incoming polarization was controlled by two polarization controllers before and after the fifth amplifier (Amp5 in Fig. 3). To ensure efficient SHG, the PPSF was first directly connected to the first amplifier (Amp1) operating at a CW power of $30 \mathrm{~mW}$ and the wavelength from the tuneable seed laser was changed gradually to see the change in the PPSF output. Once the wavelength which produces the highest SH signal is determined, the central wavelengths of both spectral filters were adjusted accordingly and the MOPA chain was reconnected. A pump power of $300 \mathrm{~mW}$ was employed at the MOPA output in order to avoid pump depletion and any other undesirable nonlinear effects. At this power level, the OSNR of the MOPA output is more than $50 \mathrm{~dB}$, suitable for relatively efficient SHG, producing an average output power of more than $3 \mathrm{~mW}$ at $775 \mathrm{~nm}$. This resulted in both the FF and
SH wavelengths existing at the output end of the PPSF. A byproduct of the SHG process, however, is the generation of a signal at the TH wavelength, generated by non-phase matched sum frequency generation (SFG), where $\omega_{3}=\omega_{1}+\omega_{2}$. This is similar to the results in [19], and can be explained by the fact that the PPSF is not perfect and the grating width contains slight fluctuations. The efficiency of this process is exceedingly small $\left(\eta \sim 10^{-9}\right)$ as the PPSF was optimized for SHG and not for the TH wavelength, but it provides a 'seed' laser with which parametric amplification can occur.

The simultaneous generation of the SH and TH wavelengths, in addition to the $\mathrm{FF}$ wavelength, allows the frequency requirement for parametric amplification via degenerate FWM as detailed in scheme (A) of Table 1 to be automatically satisfied. This is further enhanced as the FF, SH and TH are co-polarized and overlap spatially, both of which are a consequence of the SHG and SFG processes in the PPSF. Very long pulses were employed in order to eliminate walk-off effects. The resulting output spectrum from the PPSF which has been passed through a shortpass filter before tapering is given in Fig. 4.

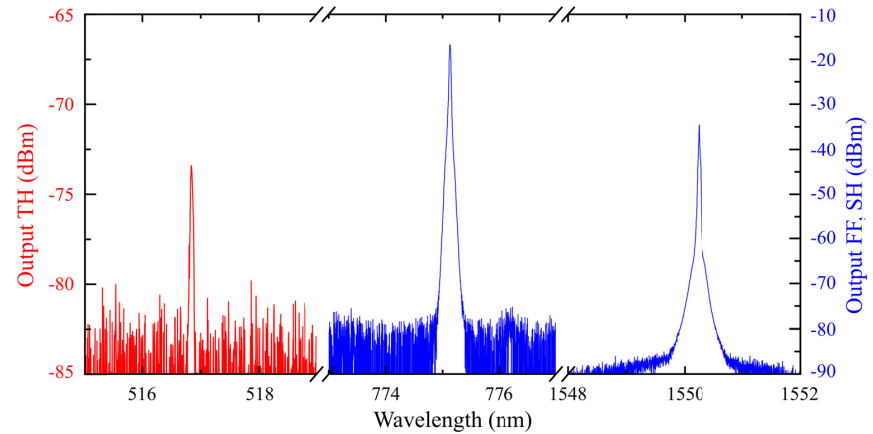

Fig. 4. Initial output signal from the PPSF after a shortpass filter. The loss of the filter is wavelength dependent, with longer wavelengths registering higher losses, causing the signal at FF (1550.3) $\mathrm{nm}$ to appear smaller than the $\mathrm{SH}(755.15 \mathrm{~nm})$, both shown here in blue. The idler signal $(516.75 \mathrm{~nm})$, shown in red, is relatively small and is just above the noise level. This is the signal which will be enhanced by the OMF, as shown in Fig. 5.

The output from the PPSF was then spliced to a length of Z-Fiber (Sumitomo Electric) before being connected with a shortpass filter, designed to have a loss of more than $15 \mathrm{~dB}$ at $775 \mathrm{~nm}$, and a broadband optical spectrum analyzer (OSA) (Yokogawa AQ6315A). The Z-Fiber is then tapered by using the modified flame brushing technique, which employs a microheater, with the tapering profile being carefully controlled in order to satisfy the adiabaticity criteria, thereby minimizing loss [7, 8]. A length of $6 \mathrm{~mm}$ of the Z-Fiber was tapered, from an initial diameter of $125 \mu \mathrm{m}$ to a final diameter of $0.5 \mu \mathrm{m}$, in order to fully explore the entire range of the predicted phase matching diameters. At the end of the tapering process, the OMF has a full length of approximately $29 \mathrm{~mm}$, with a waist length of $6 \mathrm{~mm}$ and a waist diameter of $0.5 \mu \mathrm{m}$. The $1.55 \mu \mathrm{m}$ MOPA source, connected to the PPSF, was launched into the fiber whist being tapered. The temporal evolution of the output spectra at the peak of the idler wavelength (516.7 nm) was recorded with the OSA as shown in Fig. 5. A resolution of $10 \mathrm{~nm}$ was employed to capture all signal power generated at this wavelength.

Initially, there is a very small signal at the idler wavelength, as explained above. This signal does not vary significantly as tapering takes place initially, with the small variation being accounted for by the tapering process itself as well as the thermal drift of the PPSF. As the OMF diameter reaches the first phase matching diameter $\left(d_{1} \approx 2.89 \mu \mathrm{m}\right)$, the output intensity increases by more than $22 \mathrm{~dB}$. The signal then is reduced as the fiber is tapered further, as the 
tapering process reduces the OMF length at which the phase matching diameter occurs, although due to the increasing overall length of the microfiber there is a time-varying range of diameters in the $\mathrm{OMF}$ transition region which are phase matched and nearly phase matched, resulting in a gradual decrease in the signal. This downward trend is observed until the fiber reaches the second phase matching diameter $\left(d_{1} \approx 0.80 \mu \mathrm{m}\right)$, where the signal increases dramatically by approximately $15 \mathrm{~dB}$, reaching the highest recorded output power. As the fiber is tapered even more, the signal monotonically decreases until the tapering process stops at a diameter of $0.5 \mu \mathrm{m}$. The slight drop in intensity observed at $t=580 \mathrm{~s}$ and at the end of the tapering process are due to the OMF moving inside the microheater and approaching its walls. Additionally, at the end of processing there are changes in the effective refractive index associate to the temperature change when the taper is taken out of the microheater. The inset in Fig. 5 shows the $12 \mathrm{~dB}$ increase in idler output between the untapered fiber and the OMF with $0.5 \mu \mathrm{m}$ diameter. Fig. 5 indicates that if the tapering process is stopped at an OMF with length of $6 \mathrm{~mm}$ and waist of approximately $800 \mathrm{~nm}$, a total maximum enhancement of more than $20 \mathrm{~dB}$ from the original signal can be achieved, with the figure being closer to $25 \mathrm{~dB}$ if we use the output intensity at $5 \mu \mathrm{m}$ as a baseline.

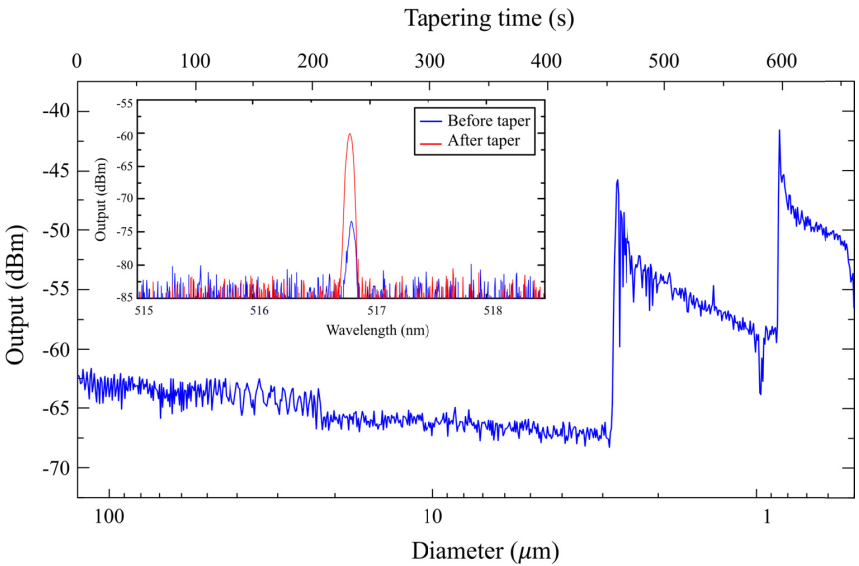

Fig. 5. Evolution of the idler signal at $516.75 \mathrm{~nm}$ with respect to the diameter and the processing time during tapering. The difference in scale between the tapering time axis (linear) and the diameter axis (logarithmic) arises from the exponential profile of the taper transition regions. Inset: the idler signal before and after the tapering process. The data in the inset was measured with a resolution of $0.05 \mathrm{~nm}$ and a higher sensitivity, as compared to $10 \mathrm{~nm}$ and a lower sensitivity for the main figure, resulting in the apparent discrepancy in the initial power measurement.

The final efficiency was increased from $\eta \sim 10^{-9}$ to $\eta \sim 10^{-7}$, corresponding to a parametric gain of $25 \mathrm{~dB}$ or $4.17 \mathrm{~dB} / \mathrm{mm}$, by using this method. This is very low compared to devices such as semiconductor optical amplifiers, where FWM efficiencies in excess of $20 \mathrm{~dB}$ are possible [20], but is comparable to other parametric amplifiers and is able to potentially operate across the entire optical bandwidth [1-7]. The final efficiency can be improved by increasing the pump-to-signal ratio, while the overall parametric gain can be improved by the fabrication of a longer OMF and by increasing the efficiency of the SHG, where an efficiency of $45 \%$ has been shown to be achievable [21].

In summary, we have demonstrated parametric amplification via FWM in optical microfibers. The amplification is achieved by tailoring the dispersion of the OMF by changing the diameter of the OMF to satisfy the phase matching requirement. An amplification of more than $20 \mathrm{~dB}$ from in the idler signal is achieved by using an OMF with a waist length of $6 \mathrm{~mm}$ seeded by a MOPA operating in the telecom C-band in conjunction with a PPSF which generates the SH wavelength required for FWM. Accounting for thermal drift of the PPSF, the total maximum FWM enhancement in the OMF is approximately $25 \mathrm{~dB}$ over a wavelength range of $1000 \mathrm{~nm}$. In principle, this technique can be extended to any wavelength, allowing for the relatively efficient generation and amplification of arbitrary wavelengths, assuming that other nonlinear effects such as SPM and XPM do not dominate.

Funding. Engineering and Physical Sciences Research Council (EPSRC) (EP/L01243X/1).

Acknowledgment. The authors would like to thank Oleksandr Tarasenko and Walter Margulis from Acreo Fiberlab who manufactured the fiber used for the PPSF as well as Peter Kazansky, Francesca Parmigiani, James Wilkinson and Senthil Ganapathy at the Optoelectronics Research Centre of the University of Southampton for access to the necessary technical facilities.

\section{References}

1. J. Hansryd, P. Andrekson, M. Westlund, J. Li, and P. Hedekvist, IEEE J. Sel. Top. Quantum Electron. 8, 506-520 (2002)

2. L. Provino, A. Mussot, E. Lantz, T. Sylvestre, and H. Maillotte, J. Opt. Soc. Am. B 20, 1532-1537 (2003)

3. J.E. Sharping, J. Lightwave Technol. 26, 2184-2191 (2008)

4. T. Torounidis, P. A. Andrekson, and B. Olsson, IEEE Photonics Technol. Lett. 18, 1194-1196 (2006)

5. G. P. Agrawal, Nonlinear Fiber Optics, (Academic, San Diego, 2013) p. 397 456.

6. J.C. Boggio, S. Moro, B. P. P. Kuo, N. Alic, B. Stossel, and S. Radic, J. Lightwave Technol. 28, 443-447 (2010)

7. A.C. Sodré, J. M. Chavez Boggio, A. A. Rieznik, H. E. Hernandez-Figueroa, H. L. Fragnito, and J. C. Knight, Opt. Express 16, 2816-2828 (2008)

8. G. Brambilla, J. Opt. 12, 043001 (2010)

9. T. Lee, Y. Jung, C. A. Codemard, M. Ding, N.G.R. Broderick, and G. Brambilla, Opt. Express 20, 8503-8511 (2012)

10. V. Grubsky, and A. Savchenko, Opt. Express 13, 6798-6806 (2005)

11. M.I.M. Abdul Khudus, T. Lee, T. Huang, X. Shao, P. Shum, and G. Brambilla, Fiber Integr. Opt. 34, 53-65 (2015)

12. M. Abdul Khudus, F. De Lucia, C. Corbari, T. Lee, P. Horak, P. Sazio, and G. Brambilla, "Enhancement of four-wave mixing frequency generation in optical microfibers [dataset]" (University of Southampton, 2015), http://dx.doi.org/10.5258/SOTON/383708

13. M. Fokine, L.E. Nilsson, Å. Claesson, D. Berlemont, L. Kjellberg, L. Krummenacher, and W. Margulis, Opt. Lett 27, 1643-1645 (2002)

14. W. Margulis, O. Tarasenko and N. Myrén, Opt. Express 17, 15534-15540 (2009)

15. R. A. Myers, N. Mukherjee, and S.R.J. Brueck, Opt. Lett. 16, 1732-1734 (1991)

16. P. G. Kazansky, and P. St J. Russel, Opt. Commun. 110, 611-614 (1994)

17. F. De Lucia, D. Huang, C. Corbari, N. Healy, and P. J. A. Sazio, Opt. Lett. 39, 6513-6516 (2014)

18. C. Corbari, A. V. Gladyshev, L. Lago, M. Ibsen, Y. Hernandez, and P. G. Kazansky, Opt. Lett. 39, 6505-6508 (2014)

19. M. Robles-Agudo, R.S. Cudney, and L. A. Ríos, Opt. Express 14, 1066310668 (2006)

20. P. P. Baveja, D. N. Maywar, and G. P. Agrawal, IEEE J. Sel. Top. Quantum Electron. 18, 899-908 (2012).

21. E. L. Lim, C. Corbari, A. V. Gladyshev, S. U. Alam, M. Ibsen, and D. J. Richardson, in Specialty Optical Fibers, (Optical Society of America, 2014), p. JTu6A-5. 


\section{References}

1. J. Hansryd, P. Andrekson, M. Westlund, J. Li, and P. Hedekvist. "Fiber-based optical parametric amplifiers and their applications." IEEE Journal of Selected Topics in Quantum Electronics 8, no. 3 (2002): 506-520.

2. L. Provino, A. Mussot, E. Lantz, T. Sylvestre, and H. Maillotte. "Broadband and flat parametric amplifiers with a multisection dispersion-tailored nonlinear fiber arrangement." JOSA B 20, no. 7 (2003): 1532-1537.

3. J.E. Sharping, "Microstructure fiber based optical parametric oscillators." Journal of Lightwave Technology 26, 14 (2008) : 2184-2191

4. T. Torounidis, P. A. Andrekson, and B. Olsson. "Fiber-optical parametric amplifier with 70-dB gain." IEEE Photonics Technology Letters 18, no. 10 (2006): 1194-1196.

5. G. P. Agrawal, Nonlinear Fiber Optics, 5th ed., Academic Press San Diego, CA: Academic, 2013, ch. 10, p. 397 - 456.

6. J.C. Boggio, S. Moro, B. P. P. Kuo, N. Alic, B. Stossel, and S. Radic. "Tunable parametric all-fiber short-wavelength IR transmitter." Journal of Lightwave Technology 28, no. 4 (2010): 443-447.

7. A.C. Sodré, J. M. Chavez Boggio, A. A. Rieznik, H. E. Hernandez-Figueroa, H. L. Fragnito, and J. C. Knight. "Highly efficient generation of broadband cascaded four-wave mixing products." Optics Express 16, no. 4 (2008): 2816-2828.

8. G. Brambilla, "Optical fibre nanowires and microwires: a review." Journal of Optics 12, no. 4 (2010): 043001.

9. T. Lee, Y. Jung, C. A. Codemard, M. Ding, N.G.R. Broderick, and G. Brambilla, "Broadband third harmonic generation in tapered silica fibres." Optics Express 20, no. 8 (2012): 8503-8511.

10. V. Grubsky, and A. Savchenko. "Glass micro-fibers for efficient third harmonic generation." Optics Express 13, no. 18 (2005): 6798-6806.

11. M.I.M. Abdul Khudus, T. Lee, T. Huang, X. Shao, P. Shum, and G. Brambilla. "Harmonic generation via $\chi 3$ intermodal phase matching in microfibers." Fiber and Integrated Optics 34, no. 1-2 (2015): 53-65.

12. M. Abdul Khudus, F. De Lucia, C. Corbari, T. Lee, P. Horak, P. Sazio, and G. Brambilla, "Enhancement of four-wave mixing frequency generation in optical microfibers [dataset]" (University of Southampton, 2015), http://dx.doi.org/10.5258/SOTON/383708.

13. M. Fokine, L.E. Nilsson, Å. Claesson, D. Berlemont, L. Kjellberg, L. Krummenacher, and W. Margulis. "Integrated fiber Mach-Zehnder interferometer for electro-optic switching." Optics Letters 27, no. 18 (2002): 1643-1645.

14. W. Margulis, O. Tarasenko and N. Myrén, "Who needs a cathode? Creating a second-order nonlinearity by charging glass fiber with two anodes", Optics Express 17, no. 18 (2009): 15534-15540.

15. R. A. Myers, N. Mukherjee, and S.R.J. Brueck. "Large second-order nonlinearity in poled fused silica." Optics Letters 16, no. 22 (1991): 17321734.

16. P.G. Kazansky, and P. St J. Russel. "Thermally poled glass: frozen-in electric field or oriented dipoles?" Optics Communications 110, no. 5 (1994): 611-614.

17. F. De Lucia, D. Huang, C. Corbari, N. Healy, and P. J. A. Sazio. "Optical fiber poling by induction." Optics Letters 39, no. 22 (2014): 6513-6516.

18. C. Corbari, A. V. Gladyshev, L. Lago, M. Ibsen, Y. Hernandez, and P. G. Kazansky, "All-fiber frequency-doubled visible laser", Optics Letters 39, no. 22 (2014): 6505-6508.

19. M. Robles-Agudo, R.S. Cudney, and L. A. Ríos. "RGB source based on simultaneous quasi-phasematched second and third harmonic generation 